Research Article

\title{
The Effects of Landscape Change on Plant Diversity and Structure in the Bale Mountains National Park, Southeastern Ethiopia
}

\author{
Annissa Muhammed $\mathbb{D}^{1,2}$ and Eyasu Elias ${ }^{1}$ \\ ${ }^{1}$ Centre for Environmental Science, Addis Ababa University, P.O.Box: 1176, Addis Ababa, Ethiopia \\ ${ }^{2}$ Department of Natural Resource Management, Arsi University, P.O.Box: 193, Assela, Ethiopia
}

Correspondence should be addressed to Annissa Muhammed; annissamuhammed@gmail.com

Received 22 November 2020; Revised 19 February 2021; Accepted 1 March 2021; Published 9 March 2021

Academic Editor: Isabel Marques

Copyright (C) 2021 Annissa Muhammed and Eyasu Elias. This is an open access article distributed under the Creative Commons Attribution License, which permits unrestricted use, distribution, and reproduction in any medium, provided the original work is properly cited.

\begin{abstract}
Bale Mountains National Park is one of the protected areas in Ethiopia that holds the largest area of Afroalpine habitat in Africa and the second largest stand of moist tropical forest. Nevertheless, human settlements, overgrazing, and recurrent fire are the main problems in the park. This study aimed to determine the effects of human-induced landscape change in floristic composition and structure in the park. The vegetation data were collected systematically from 96 sample plots laid along 24 line transects in the edge and interior habitats of the six land cover types. Vegetation composition and landscape structural analysis were made using $R$ software version 3.5.2 and FRAGSTATS version 4.2.1, respectively. Patch number was strong and positively affected species richness $(r=-0.90, p<0.05)$, diversity $(r=-0.96, p<0.01)$, and basal area $(r=-0.96, p<0.001)$, whereas mean patch size was strong and negatively influenced species richness $(r=0.95, p<0.05)$, diversity $(r=0.87, p<0.05)$, and basal area $(r=0.82$, $p<0.05)$. The overall species richness, Shannon diversity index, and Margalef index were significantly higher in the edge habitat; however, the mean basal area of woody species was significantly higher in the interior habitat at $p<0.05$. This study uncovered that the park is floristically rich and diverse, and it provides a variety of ecological and economic benefits to the surrounding community and to the nation at large. However, these benefits are gradually declining due to the high level of anthropogenic activities in the park. Thus, integrated environmental management strategy that blends with sustainable use of natural resources should be implemented to minimize the threats.
\end{abstract}

\section{Introduction}

Landscapes all over the world are alarmingly changed and fragmented due to anthropogenic factors such as urbanization, agricultural expansion, forest fire, and climate change $[1,2]$. Most of the global changes responsible for the reduction in population and biodiversity are exacerbated by fragmentation $[3,4]$. The primary causes of global biodiversity reduction are the destruction and degradation of natural ecosystems [5]. Predominantly, habitat loss and fragmentation are presently the main threats to terrestrial biodiversity [6]. Moreover, habitat fragmentation can affect the species interactions and community composition, as invasive or pest species, and may substitute the original species pool and increase the transmission and prevalence of the disease in small fragments [7]. Moreover, the species richness and abundance usually decrease with reduced patch size [8]. As landscapes become more fragmented, patch diversity increases with subsequent increase in the edge, exotic, and generalist species and ultimately leads to the reduction in landscape quality as habitat for species [9]. Accordingly, species richness in interior habitat, particularly indigenous and specialist species, tends to decrease [10]. The number of species existing in a patch tends to rise with patch size up to a certain limit, and the types of species found also tend to vary in size [8]. Size and shape interact to influence 
the amount of interior area remaining in a particular habitat fragment [2].

Tropical montane ecosystem is one of the hot spot ecosystems on Earth that comprises more than 200,000 species of flowering plants $[11,12]$. The Ethiopian highland, which is located in the tropical region, encompasses over $50 \%$ of the Afromontane vegetation in Africa [13]. A suitable geographical position, a wide range of altitude, a high amount of rainfall, and a wide range of temperature variations equip the area with huge ecological diversity and a wealth of biological resources [14]. However, severe deforestation coupled with the cultivation of steep marginal lands, overgrazing, and sociopolitical uncertainty has resulted in rigorous land degradation over large areas of the country [15]. The overdependence of the country's economy on agricultural production and the existence of more than $80 \%$ of the population in the highlands [16, 17] mainly contribute to the degradation of ecological resources and biodiversity loss.

The mountainous landscape and the mosaic of natural vegetation in the Bale Mountains have considerable economic, recreational, esthetic, and scientific importance [14]. The Bale Mountains National Park (BMNP) is the most significant conservation area situated in this region of Ethiopia and established in 1969 to preserve the endemic and indigenous floras and faunas in the area $[18,19]$. It is one of the 34 International Biodiversity Hotspots and meets the requirements for the World Heritage Site and Biosphere Reserve Listing [20]. However, the park is facing a critical challenge from the illegal settlement and overgrazing and that leads to the change in its landscape structure and function. As a result, the habitats in the park are changing and the provision of ecological services from it is substantially reduced. Consequently, no research provides detailed information about the landscape structure and its potential impact on vegetation composition and structure in the park. Therefore, this research was aimed to analyze the potential impact of landscape change in floristic composition, diversity, and structure in the BMNP. Particularly, a comparative analysis was made among the edge and interior habitats of the park.

\section{Materials and Methods}

2.1. Study Area Description. BMNP is located within the geographic bounds of $6^{\circ} 53^{\prime} 08^{\prime \prime} \mathrm{N}$ latitude and $39^{\circ} 44^{\prime} 03^{\prime \prime} \mathrm{E}$ longitude and $400 \mathrm{~km}$ southeast of Addis Ababa, Ethiopia (Figure 1). It comprehends a wide range of habitats between $1450 \mathrm{~m}$ and $4377 \mathrm{~m}$ altitude. The park holds the largest area of Afroalpine habitat (about $1000 \mathrm{~km}^{2}$ ) above $3000 \mathrm{~m}$ asl in Africa and the second largest stand of moist tropical forest [21]. It is one of the 34 International Biodiversity Hotspots and also qualifies for World Heritage Site and Biosphere Reserve Listing [22]. It received rainfall that ranged from 520 to $2370 \mathrm{~mm}$ annually [23], and the distributional pattern is bimodal with heavy rains from July to October (highest peak in August) and small rains from March to June (highest peak in April). The mean monthly minimum and maximum temperatures are $5.6^{\circ} \mathrm{C}$ and $21.4^{\circ} \mathrm{C}$, respectively. Its soil is fertile silty loam of reddish-brown to black clay soils dominated by Vertic Cambisols and Leptosols [24].

2.2. Vegetation Sampling Design. From 13 to 20 November 2018, a reconnaissance survey was conducted to get insights into the vegetation physiognomy and establish sampling sites in the study area. Following, the actual fieldwork was performed in the dry season between November 2019 and January 2020. A total of 96 sample plots $(20 \times 20 \mathrm{~m})$ were systematically laid along 24 line transects in eight directions along three altitudinal gradients at $100 \mathrm{~m}$ elevational differences as it maximizes the distance between plots and minimizes spatial correlation among the observations [25]. To make a comparison between the vegetation data, an equal number of sample plots have been laid on the edge and interior habitats following Daye [26].

2.3. Species Identification. Plant species in the nested plots were identified at the field with the help of local peoples (for vernacular names) and by referring different volumes of Flora of Ethiopia and Eritrea books [27, 28]. For the species that were difficult to identify in the field, representative specimens were cut, numbered, and pressed at the site. The collections were named using folk taxonomy, and identification of formal taxonomy was determined using the voucher specimens at the National Herbarium, Addis Ababa University.

2.4. Floristic Composition and Structure. The most commonly used diversity indices of species richness $(S)$, Simpson index $(D)$, Shannon-Wiener index $\left(H^{\prime}\right)$, Pielou's evenness index $\left(J^{\prime}\right)$, Whittaker $\beta$-diversity $\left(\beta_{w}\right)$, Margalef index $\left(D_{\mathrm{M}}\right)$, and Berger-Parker index $(d)$ were computed to analyze the patterns of plant diversity in the edge and interior habitats following Magurran [29] and Økland [30] using equations (1)-(4):

$$
H^{\prime}=-\sum_{i=1}^{s} P_{i} \ln P_{i}
$$

where $H^{\prime}$ is the Shannon diversity index, $P_{i}$ is the proportion of individuals, and $\ln$ is the natural logarithm.

$$
J=\frac{H^{\prime}}{H_{\max }},
$$

where $H_{\max }$ is the maximum level of diversity possible within a given population $(\ln S)$ and $S$ is species richness.

$$
\beta-\text { diversity }=\frac{a+c}{2 a+b+c}
$$

where $a$ is the number of shared species in two sites and $b$ and $c$ are the numbers of species unique to each site.

The Margalef diversity index $\left(D_{M}\right)$ was computed using the following formula: 


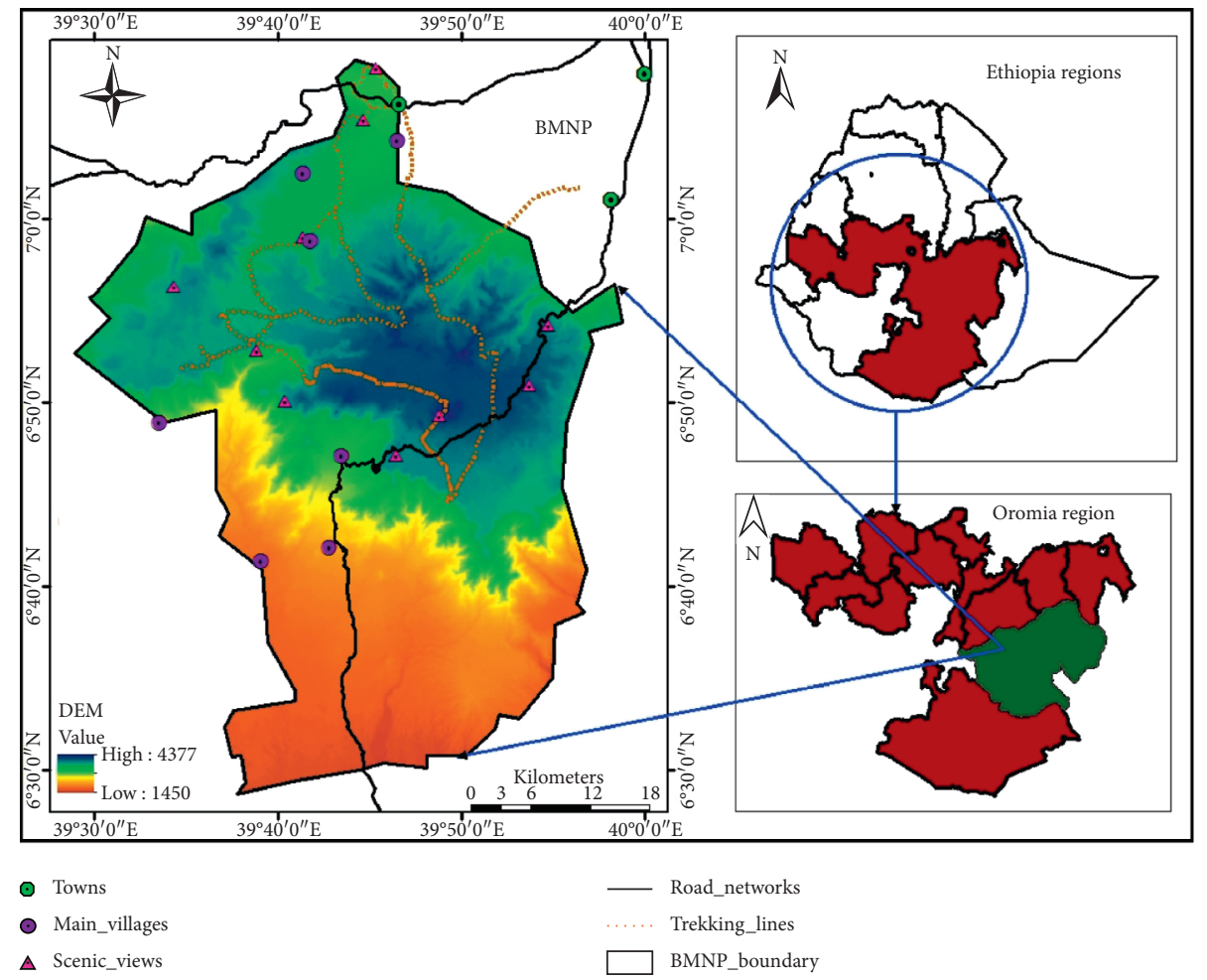

FIgURE 1: Location map of the study area.

$$
D_{M}=\frac{s-1}{\ln N}
$$

where $D_{M}$ is the Margalef diversity index, $S$ is the number of species, and $N$ is the total number of individuals in the sample.

The woody species density, frequency, dominance, and their relative values in the interior and edge habitats were computed to obtain the important value index and describe the woody species structure following Ellenberg and Mueller-Dombois [31] and Martin [32] using equations (5)-(8). Moreover, $\mathrm{DBH}$, tree height, and basal area were analyzed to determine the population structure following Kitessa et al. [33] and Van der Maarel [34]:

$$
\mathrm{BA}=\frac{\pi d^{2}}{4}
$$

where BA is the basal area, $\pi=3.14$, and $d$ is the $\mathrm{DBH}(\mathrm{cm})$.

$$
\mathrm{Fr}=\frac{P_{i}}{\sum_{i=1}^{s} P_{i}} \times 100,
$$

where Fr is the frequency of a species and $P_{i}$ is the number of plots in which the $i^{\text {th }}$ species occurred.

$$
R_{\mathrm{de}}=\frac{n_{i}}{\sum_{i=1}^{s} n_{i}} \times 100
$$

where $R_{\mathrm{de}}$ is the relative density and $n_{i}$ is the number of individuals of the $i^{\text {th }}$ species.

$$
\mathrm{IVI}=R_{\mathrm{de}}+R_{\mathrm{Fr}}+R_{\mathrm{Do}}
$$

where IVI is the importance value index, $R_{\mathrm{de}}$ is the relative density, $R_{\mathrm{Fr}}$ is the relative frequency, and $R_{\mathrm{Do}}$ is the relative dominance.

2.5. Measurement of Landscape Structure. Landsat images of the years 1985, 1995, 2005, and 2017 were processed using ArcGIS version 10.3 to produce time-series datasets of land use/land cover. Then, eight landscape indices were analyzed using the processed land use/land cover data following McGarigal et al. [35] and Smiraglia et al. [36]. The indices include patch number (PN), mean patch size (AREA_MN), total core area (TCA), edge density (ED), area-weighted mean shape index (SHAPE_AM), mean Euclidean nearest neighbor distance (ENN_MN), and interspersion and juxtaposition index (IJI). Edge habitat was identified by deducting $50 \mathrm{~m}$ from the edge of each vegetation type. FRAGSTATS software version 4.2.1 was used to compute the landscape patterns in each land cover class and the entire landscape [37]. The twoway analysis of variance (two-way ANOVA) and linear regression analysis were made to test significant differences between fragmentation indices and species composition and structure parameters following the post hoc Tukey's highly significance difference (Tukey's HSD) test at 5\% significance level using PAST software version 4.02 [38].

\section{Results and Discussion}

3.1. Landscape Structure Change. The analysis of landscape structure in this study revealed that the habitats in the BMNP are progressively transformed. The area has shown an increase in PN by $40.2 \%$ and a decrease in AREA_MN by 
28.7\% from 1985 to 2017. According to Oertli et al. [39], the high number of separated patches in a habitat indicates a high level of fragmentation. Across the entire study period, SHAPE_AM, which indicates the complexity of patch shape, increased by $18.8 \%$. A higher perimeter-area relationship characterizes the rapid rate of fragmentation in the landscape [40]. Moreover, there was inconsistency in the values of ED; however, it was increased by $22.3 \%$ over the study period. As it was emphasized by McGarigal [37], the oscillation of ED indicated a major reduction in the spatial heterogeneity of the landscape. Conversely, the study area has shown a declining trend in TCA by $10.6 \%$ from 1985 to 2017. This was due to the escalated level of disturbances in the study area. As it was reported by Kidane et al. [41], the most dominant practices in the Bale Mountains, especially after 1995, were the upward expansion of agriculture and enrichment plantation.

The isolation of patches within the landscape of the study area was increased from $105.22 \mathrm{~m}$ to $111.94 \mathrm{~m}$ overtime (Table 1). This result is in agreement with the result reported by Tolessa et al. [42] in the central highlands of Ethiopia and Daye [26] in Southwest Ethiopia. Conversely, the intermixing of patches in the study area showed an overall declining trend from 95.38 to 86.77 over the study period. This result showed that the BMNP constitutes more scattered patches compared to other similar areas studied by Posada Posada [43] and Tolessa et al. [42].

3.2. Overall Floristic Composition and Structure. A total of 205 plant species belonging to 71 families and 153 genera were recorded (Table 2). Of these, 50 species were trees, 52 were shrubs, 12 were lianas, and 91 were herbs. Asteraceae was the most dominant family with 31 species, followed by Fabaceae with 11 species. Conversely, Helichrysum was the most abundant genus with 9 species, followed by Alchemilla and Trifolium with 5 species each. Twenty endemic species, including Euphorbia dumalis S. Carter, Lobelia rhynchopetalum Hemsl., and Thymus schimperi subsp. Schimperi Ronniger was identified in this study. The overall Shannon diversity and evenness index of the study area were 4.34 and 0.81 , respectively. This indicated that the study area was more diverse compared to other similar vegetation areas including Bonga forest [44], Agama forest [45], and Munessa forest [13]. Conversely, the total density of seedlings, saplings, and mature trees in the study area was 8751, 4413, and 1567 individuals $\mathrm{ha}^{-1}$, respectively. This was lower than other comparable areas such as Kuandisha forest [46] and Wof-Washa forest [47]. The ratios of seedling to mature tree, sapling to mature tree, and seedling to sapling were 5.58, 2.82, and 1.98, respectively. This shows the recruitment potential of the forest is relatively higher [48].

Woody species density with $\mathrm{DBH}^{\circ}>02 \mathrm{~cm}$ was 1567 individuals $\mathrm{ha}^{-1}$. This was relatively higher compared to other similar vegetation areas such as the Wof-Washa forest [48] and Agama forest [45]. The most frequent woody species was Croton macrostachyus Hochst. ex Del with $81 \%$ frequency followed by Juniperus procera L. (79\%), Podocarpus falcatus (Thunb) C.N (63\%), and Hagenia abyssinica (Bruce) J.F.
Gmel (60\%). Conversely, the total basal area of woody species was $170.26 \mathrm{~m}^{2} \mathrm{ha}^{-1}$, and it was considerably higher compared to other similar vegetation areas in Ethiopia. About $75 \%$ of the basal area was contributed by five tree species such as Juniperus procera $\left(46.71 \mathrm{~m}^{2} \mathrm{ha}^{-1}\right)$, Syzygium guineense (Willd.) DC $\left(24.76 \mathrm{~m}^{2} \mathrm{ha}^{-1}\right)$, Cordia africana Lam $\left(20.95 \mathrm{~m}^{2} \mathrm{ha}^{-1}\right)$, Hagenia abyssinica $\left(18.47 \mathrm{~m}^{2} \mathrm{ha}^{-1}\right)$, and Ehretia cymose Thonn $\left(15.86 \mathrm{~m}^{2} \mathrm{ha}^{-1}\right)$. Consequently, Juniperus procera was the dominant woody species with an IVI of 26.43. The species with higher IVI values in the study area was among the characteristic species in similar vegetation types in Ethiopia [49, 50].

3.3. Floristic Composition and Structure in the Edge and Interior Habitat. A total of 136 species belonging to 111 genera and 59 families were identified in the edge habitats of the sampled patches, whereas 117 species that belonging to 84 genera and 40 families were recorded in the interior habitats. From the identified life forms, 19 species were trees, 22 species were shrubs, 86 species were herbs, and 7 species were lianas in the edge habitats, whereas 28 species were trees, 21 species were shrubs, 57 species were herbs, and 11 species were lianas in the interior habitats. The overall means $( \pm \mathrm{SE})$ species richness $(35 \pm 4.2)$, Shannon diversity index $(2.93 \pm 0.17)$, and Margalef index $(5.68 \pm 0.69)$ of the edge habitat were significantly higher compared to the interior habitat at $p<0.05$ (Table 3 ). These variations could be due to the differences in site productivity, habitat heterogeneity, and disturbance factors $[44,51]$ or the invasion of exotic plant species [52]. However, the woody species richness in the interior habitat (28) was significantly higher than the edge (17). Moreover, the evenness index in the interior habitats $(0.83 \pm 0.04)$ was higher, but not significant, than the edge habitat $(0.79 \pm 0.05)$. This result was in agreement with the finding in [53]. Abiotic factors, seed predation, loss of pollinators and seed dispersers, and tree mortality were reported as the common causes for the differences in woody species composition between the edge and interior habitats $[53,54]$. The computed Sorensen's similarity index depicted that the number of species in the edge habitats was $45 \%$ similar to the species in the interior habitats. This value indicated that the similarity between the edge and interior habitat was weak [13]. The mean density of seedling $\left(995.42 \pm 19.27\right.$ individuals $\left.\mathrm{ha}^{-1}\right)$, sapling $(509.29 \pm 9.06$ individuals $\left.\mathrm{ha}^{-1}\right)$, and mature trees $(187.60 \pm 4.70$ individuals $\mathrm{ha}^{-1}$ ) in the interior habitat was significantly higher compared to the edge. This indicates that the recruitment potential of the interior forest was significantly higher compared to the edge habitat [48]. This could be due to the increased mortality rates of seedling, sapling, and mature trees in the edge habitats $[53,55]$.

The mean woody species density in the interior habitat $\left(85 \pm 22.17\right.$ stems $\left.^{-1} \mathrm{a}^{-1}\right)$ was significantly higher compared to the edge habitat $\left(70 \pm 16.53\right.$ stems ha $\left.\mathrm{ha}^{-1}\right)$ at $p<0.05$ (Figure 2(a); Tables 4 and 5). This could be due to the selective cutting of trees for timber production, house construction, and firewood in the edge habitats, which ultimately leads to a reduction in the density of large trees 
TABLE 1: Landscape structural characteristic of the BMNP from 1985 to 2017.

\begin{tabular}{lccccccc}
\hline Year & NP & AREA_MN (ha) & SHAPE_AM & TCA $\left(\mathrm{km}^{2}\right)$ & ED $\left(\mathrm{m} / \mathrm{m}^{2}\right)$ & ENN_MN $(\mathrm{m})$ & IJI $(\%)$ \\
\hline 1985 & 25864 & 8.42 & 24.97 & 1568.91 & 60.53 & 107.27 \\
1995 & 30582 & 7.12 & 24.12 & 1489.10 & 69.18 & 105.22 \\
2005 & 29329 & 7.42 & 29.36 & 1471.02 & 66.44 & 71.69 \\
2017 & 36267 & 6.00 & 29.67 & 1402.59 & 74.02 & 79.29 \\
$\%$ & 40.22 & -28.68 & 18.83 & -10.60 & 22.28 & 109.21 \\
\hline
\end{tabular}

Note. The negative sign of percentage implies a decreasing trend, and the positive sign implies an increasing trend.

TABLE 2: List of plant species identified in the BMNP.

\begin{tabular}{|c|c|c|c|c|}
\hline Scientific name & Family & Local name (Or.) & Habit & Coll. no. \\
\hline Acacia oerfota (Forssk.) Schweinf. & Fabaceae & Wanga & $\mathrm{S}$ & AM168 \\
\hline Acacia senegal (L.) Willd. & Celastraceae & Karxafa & $\mathrm{S}$ & AM172 \\
\hline Achyranthes aspera $\mathrm{L}$. & Amaranthaceae & Roppe, Qorsa Waranssa & $\mathrm{H}$ & AM094 \\
\hline Agrostis sclerophylla C.E. Hubb. & Poaceae & Mergeseri & $\mathrm{H}$ & AM009 \\
\hline Ajuga bracteosa Wall. ex Benth. in Wall. & Lamiaceae & - & $\mathrm{H}$ & AM078 \\
\hline Albizia gummifera (J. F. Gmel.) C.A.Sm. & Mimosaceae & Karchofe & $\mathrm{T}$ & AM174 \\
\hline Alchemilla abyssinica Fresen. & Rosaceae & Hindriff & $\mathrm{H}$ & AM043 \\
\hline Alchemilla cryptantha Steud. ex A Rich. & Rosaceae & Hindriff & $\mathrm{H}$ & AM159 \\
\hline Alchemilla haumanii Rothm. & Rosaceae & - & $\mathrm{H}$ & AM055 \\
\hline Alchemilla pedata A. Rich. & Rosaceae & Hindriff, Indriif & $\mathrm{H}$ & AM017 \\
\hline Alchemilla rothii Oliv. & Rosaceae & - & $\mathrm{H}$ & AM052 \\
\hline Alepidea peduncularis Steud. ex A. Rich. & Apiaceae & - & $\mathrm{H}$ & AM060 \\
\hline Allophyllus macrobotrys Gilg & Sapindaceae & Abara & $\mathrm{T}$ & AM131 \\
\hline Allophylus abyssinicus (Hochst.) Radlk. & Sapindaceae & Sarara & $\mathrm{T}$ & AM178 \\
\hline Anaptychia liucomeleana Wain. & Physciaceae & Lichen & $\mathrm{H}$ & AM054 \\
\hline Annona reticulata $\mathrm{L}$. & Annonaceae & Gishta & $\mathrm{T}$ & AM161 \\
\hline Anthemis tigreensis J. Gay ex A. Rich. & Asteraceae & - & $\mathrm{H}$ & AM012 \\
\hline Argemone mexicana $\mathrm{L}$. & Papaveraceae & Qore Haree & $\mathrm{H}$ & AM018 \\
\hline Artemisia afra Jacq. ex Willd. & Asteraceae & Tepenea, Tepeno & $\mathrm{H}$ & AM007 \\
\hline Asparagus africanus Lam. & Asparagaceae & Seriti & $\mathrm{S}$ & AM199 \\
\hline Asplenium aethiopicum (Burm.f.) Bech. & Aspleniaceae & Qumbuta & $\mathrm{H}$ & AM155 \\
\hline Astragalus atropilosulus (Hochst.) Bange & Fabaceae & Hara & $\mathrm{E}$ & AM037 \\
\hline Bidens macroptera (Sch. Bip. ex Chiov.) Mesfin & Asteraceae & Hade gola & $\mathrm{H}$ & AM040 \\
\hline Blyttia fruticulosum (Decne.) D.V.Field & Apocynaceae & Homba & $\mathrm{H}(\mathrm{clim})$ & AM122 \\
\hline Brachycorythis buchananii (Schltr.) Rolfe & Orchidaceae & Shumbura gala & $\mathrm{H}$ & AM066 \\
\hline Bromus pectinatus Thunb. & Poaceae & Alanmuressa & $\mathrm{H}$ & AM106 \\
\hline Calpurnia aurea (Ait.) Benth. & Fabaceae & Cheekata & S & AM167 \\
\hline Carduus leptacanthus Fresen. & Asteraceae & Qore Haree & $\mathrm{H}$ & AM107 \\
\hline Carduus nyassanus (S. Moore) R.E. Fries & Asteraceae & Qore Haree & $\mathrm{H}$ & AM033 \\
\hline Carissa edulis (Forssk.) Vahl & Apocynaceae & Hagamssa(Or), Agam(Amh) & $\mathrm{S}$ & AM181 \\
\hline Carissa spinarum L. & Apocynaceae & Harangma & S & AM200 \\
\hline Casimiroa edulis La Llave \& Lex. & Rutaceae & Kasmira & $\mathrm{T}$ & AM163 \\
\hline Catha edulis (Vahl) Forssk. ex Endl. & Celastraceae & Jimaa & S & AM143 \\
\hline Celtis africana Burm.f. & Ulmaceae & Meteqamma & $\mathrm{T}$ & AM116 \\
\hline Centella asiatica (L.) Urb. & Apiaceae & Balee, Qudu & $\mathrm{H}$ & AM064 \\
\hline Cerastium afromontanum T.C.E. Fr. \& Weim. & Caryophyllaceae & Duqusha chuffa & $\mathrm{H}$ & AM087 \\
\hline Citrus aurantifolia (Christm.) Swingle & Rutaceae & Lomii & $\mathrm{S}$ & AM186 \\
\hline Citrus sinensis (L.) Osbeck & Rutaceae & Burtukana & $\mathrm{S}$ & AM187 \\
\hline Clematis hirsuta Perr. \& Guill. & Ranunculaceae & Fitii & $\mathrm{Li}$ & AM114 \\
\hline Coffea arabica $\mathrm{L}$. & Rubiaceae & Buuna & $\mathrm{S}$ & AM121 \\
\hline Combretum ghasalense Engl. \& Diels & Combretaceae & Dhandhaasa & $\mathrm{T}$ & AM190 \\
\hline Commelina africana $\mathrm{L}$. & Commelinaceae & Gura Jarsa & $\mathrm{H}$ & AM020 \\
\hline Cordia africana Lam. & Boraginaceae & Wodessa & $\mathrm{T}$ & AM160 \\
\hline Craterostigma plantagineum Hochstetter & Scrophulariaceae & - & $\mathrm{H}$ & AM102 \\
\hline Crepis carbonaria Sch. Bip. & Asteraceae & Marga Hoffi & $\mathrm{H}$ & AM025 \\
\hline Crepis ruepellii Sch. Bip. & Asteraceae & - & $\mathrm{H}$ & AM071 \\
\hline Crotolaria agatiflora Schweinf. Sub.sp. ErlangeriBak. F. & Fabaceae & Shashamane & $\mathrm{S}$ & AM201 \\
\hline Croton macrostachyus Hochst. ex Del. & Euphorbiaceae & Makkannisa & $\mathrm{T}$ & AM119 \\
\hline Cuscuta kilimanjari Oliv. & Convolvulaceae & Segeniti & $\mathrm{H}(\mathrm{clim})$ & AM098 \\
\hline
\end{tabular}


TABle 2: Continued.

\begin{tabular}{|c|c|c|c|c|}
\hline Scientific name & Family & Local name (Or.) & Habit & Coll. no. \\
\hline Cycniopsis humifusa (Forssk.) Engl. & Scrophulariaceae & - & $\mathrm{H}$ & AM080 \\
\hline Cynoglossum amplifolium Hochst. ex DC. & Boraginaceae & Qarccabbaa & $\mathrm{H}$ & AM081 \\
\hline Cynoglossum coeruleum Hochst. & Boraginaceae & Qarccabbaa & $\mathrm{H}$ & AM026 \\
\hline Cynoglossum lanceolatum Forssk. & Boraginaceae & - & $\mathrm{H}$ & AM058 \\
\hline Cyperus schimperianus Steud. & Cyperaceae & Alando & $\mathrm{H}$ & AM023 \\
\hline Dianthoseris schimperi A. Rich & Asteraceae & - & $\mathrm{H}$ & AM056 \\
\hline Dicrocephala integrifolia (L.f.) Kuntze & Asteraceae & - & $\mathrm{H}$ & AM105 \\
\hline Diospyros abyssinica (Hiern) F. White & Ebenaceae & Lookoo & $\mathrm{T}$ & AM153 \\
\hline Diospyros mespiliformis Hochst. ex A.DC & Ebenaceae & Kolati & $\mathrm{T}$ & AM176 \\
\hline Discopodium eremanthum Chiov. & Solanaceae & Meraro & $\mathrm{S}$ & AM084 \\
\hline Dracaena afromontana Mildbr. & Dracaenaceae & Ruukeessa & $\mathrm{T}$ & AM194 \\
\hline Echinops hoehnelii Schweinf. & Asteraceae & Qore Haree & $\mathrm{S}$ & AM099 \\
\hline Echinops macrochaetus Fresen. & Asteraceae & Tuqa, Qoree & $\mathrm{H}$ & AM036 \\
\hline Ehretia cymosa Thonn. & Boraginaceae & Ulaagaa & $\mathrm{T}$ & AM135 \\
\hline Elaeodendron buchananii (Loes) Loes. & Celasteraceae & Xilloo & $\mathrm{T}$ & AM137 \\
\hline Entada abyssinica Steudel ex A. Rich. & Mimosoideae & Kontir & S & AM075 \\
\hline Erica arborea L. & Ericaceae & Satoo & $\mathrm{S} / \mathrm{T}$ & AM073 \\
\hline Erica trimera (Engl.) Beentje & Ericaceae & - & S & AM065 \\
\hline Erythrina brucei Schweinf. & Fabaceae & Waleensu & $\mathrm{T}$ & AM175 \\
\hline Euclea schimperi (A.DC.) Dandy & Ebenaceae & Miheesa & $\mathrm{T}$ & AM164 \\
\hline Euphorbia depauperata A. Rich. & Euphorbiaceae & Guri Xixiqo & $\mathrm{H}$ & AM010 \\
\hline Euphorbia dumalis S. Carter & Euphorbiaceae & Gurii & $\mathrm{S}$ & AM090 \\
\hline Eurynchium pulchellum (Hedw.) Jenn. & Brachytheciaceae & Hasufe (O), Mosses (E) & $\mathrm{E}$ & AM044 \\
\hline Euryops prostratus B. Nordenst. & Asteraceae & - & $\mathrm{S}$ & AM051 \\
\hline Fagaropsis angolensis (Engl.) Milne & Rutaceae & Siisaa & $\mathrm{T}$ & AM150 \\
\hline Ferula communis L. & Apiaceae & Gnida & $\mathrm{H}$ & AM014 \\
\hline Festuca abyssinica A.Rich. & Poaceae & - & $\mathrm{H}$ & AM062 \\
\hline Ficus vasta Forssk. & Moraceae & Qiltu & $\mathrm{T}$ & AM169 \\
\hline Filicium decipiens (Wight \& Am.) Thw. & Sapindaceae & Caanaa & $\mathrm{T}$ & AM156 \\
\hline Flacourtia indica (Burm.f.) Merr. & Salicaceae & Hokoku & $\mathrm{S}$ & AM180 \\
\hline Galium simense Fresen. & Rubiaceae & Maxxane & $\mathrm{H}$ & AM016 \\
\hline Geranium arabicum Forssk. & Geraniaceae & Bucha & $\mathrm{H}$ & AM068 \\
\hline Geranium kilimandscharicum Engl. & Geraniaceae & Balee Tiqo & $\mathrm{H}$ & AM097 \\
\hline Gouania longispicata Engl. & Rhamnaceae & Wayebossaa & $\mathrm{H}(\mathrm{clim})$ & AM128 \\
\hline Grevillea robusta A. Cunn. ex R. Br. & Proteaceae & Grevillea & $\mathrm{T}$ & AM170 \\
\hline Gynura pseudochina (L.) DC. & Asteraceae & Raffu & $\mathrm{H}$ & AM101 \\
\hline Habenaria peristyloides A. Rich. & Orchidaceae & Kerkashaw & $\mathrm{H}$ & AM112 \\
\hline Hagenia abyssinica (Bruce) J.F. Gmel. & Rosaceae & Hexxoo & $\mathrm{T}$ & AM082 \\
\hline Haplocarpha rueppellii (Sch. Bip.) Beauv. & Asteraceae & - & $\mathrm{H}$ & AM113 \\
\hline Hebenstretia angolensis Rolfe & Scrophulariaceae & - & $\mathrm{H}$ & AM104 \\
\hline Hebenstretia dentata $\mathrm{L}$. & Scrophulariaceae & - & $\mathrm{H}$ & AM032 \\
\hline Helichrysum citrispinum Del. & Asteraceae & - & $\mathrm{S}$ & AM042 \\
\hline Helichrysum foetidum (L.) Moench. & Asteraceae & - & $\mathrm{H}$ & AM011 \\
\hline Helichrysum formosissimum (Sch.Bip.) Sch.Bip. ex A.Rich. & Asteraceae & - & $\mathrm{S}$ & AM063 \\
\hline Helichrysum globosum A. Rich. & Asteraceae & - & $\mathrm{H}$ & AM024 \\
\hline Helichrysum gofense Cufod. & Asteraceae & - & $\mathrm{H}$ & AM006 \\
\hline Helichrysum harenensis Mesfin. & Asteraceae & Ufea/Hoffii & $\mathrm{H}$ & AM039 \\
\hline Helichrysum quartitianum A. Rich. & Asteraceae & Agadena & $\mathrm{H}$ & AM095 \\
\hline Helichrysum schimperi (Sch. Bip. ex A. Rich.) Moeser & Asteraceae & Badubera & $\mathrm{H}$ & AM048 \\
\hline Helichrysum splendidum (Thunb.) Less. & Asteraceae & Badubera & $\mathrm{S}$ & AM001 \\
\hline Hibiscus calyphyllus Cavan. & Malvaceae & Hincinni & $\mathrm{H}$ & AM146 \\
\hline Hippocratea africana (Willd.) Loes. & Celasteraceae & Gaguro & $\mathrm{H}(\operatorname{clim})$ & AM145 \\
\hline Hippocratea goetzei Loes & Celasteraceae & Gaalee Gaguro & $\mathrm{H}(\mathrm{clim})$ & AM152 \\
\hline Hippocratea pallens Planchon ex Oliver & Celasteraceae & Xara'a & $\mathrm{H}(\mathrm{clim})$ & AM147 \\
\hline Hydrocotyle mannii Hook.f. & Apiaceae & - & $\mathrm{H}$ & AM072 \\
\hline Hypericum peplidifolium A. Rich. & Hypericaceae & & $\mathrm{H}$ & AM035 \\
\hline Hypericum revolutum Vahl & Hypericaceae & Geremba & $\mathrm{T} / \mathrm{S}$ & AM002 \\
\hline Hypericum scioanum Chiov. & Hypericaceae & - & $\mathrm{H}$ & AM031 \\
\hline Inula confertiflora A. Rich. & Asteraceae & Haxxawii & S & AM197 \\
\hline Jasminum abyssinicum Hochst. ex Dc & Oleaceae & Dikii & $\mathrm{H}(\mathrm{clim})$ & AM123 \\
\hline
\end{tabular}


TABle 2: Continued.

\begin{tabular}{|c|c|c|c|c|}
\hline Scientific name & Family & Local name (Or.) & Habit & Coll. no. \\
\hline Juniperus procera $\mathrm{L}$. & Cupressaceae & Hindessa & $\mathrm{T}$ & AM083 \\
\hline Kalanchoe petitiana A. Rich. & Crassulaceae & - & $\mathrm{S}$ & AM103 \\
\hline Kniphofia foliosa Hochst. & Asphodelaceae & Lela & $\mathrm{H}$ & AM008 \\
\hline Kniphofia insignis Rendle & Asphodelaceae & Lela Xixiqo & $\mathrm{H}$ & AM027 \\
\hline Kniphofia isoetifolia Steud. ex Hochst. & Asphodelaceae & Lela Xixiqo & $\mathrm{H}$ & AM013 \\
\hline Landolphia buchananii (Hall.f.) Stapf & Apocynaceae & Homba & $\mathrm{H}(\mathrm{clim})$ & AM151 \\
\hline Lannea schimperi (Hochst. ex A.Rich.) Engl. & Anacardiaceae & Andarku & S & AM185 \\
\hline Leonotis ocymifolia (Burm.f.) Iwarsson & Lamiaceae & Bokolu & S & AM202 \\
\hline Lepidotrichilia volkensii (Gurke) Leroy & Meliaceae & Saakarro & $\mathrm{T}$ & AM148 \\
\hline Leucaena leucocephala (Lam.) de Wit & Mimosoideae & Lucinaa & S & AM189 \\
\hline Lobelia rhyncopetalum Hemsl. & Lobeliaceae & Taruurra(O), Jibra(Am) & $S$ & AM041 \\
\hline Macaranga capensis (Baill.) Sim & Euphorbiaceae & Argoo & $\mathrm{T}$ & AM140 \\
\hline Malva verticillata $\mathrm{L}$. & Malvaceae & Lita & $\mathrm{S}$ & AM029 \\
\hline Mangifera indica L. & Anacardiaceae & Mango & $\mathrm{T}$ & AM162 \\
\hline Margaritaria discoidea (Baill.) Webster & Phyllanthaceae & Bulala & $\mathrm{T}$ & AM141 \\
\hline Maytenus arbutifolia (A. Rich.) Wilczek & Celastraceae & Kombolcha & $\mathrm{T}$ & AM173 \\
\hline Maytenus obscura (A. Rich.) Cuf. & Celastraceae & Kombolcha, Duqusha (Or.) & $\mathrm{S}$ & AM091 \\
\hline Maytenus undata (Thunb.) Blakelock & Celastraceae & Kombolcha & $\mathrm{S}$ & AM100 \\
\hline Melia azedarach L. & Meliaceae & Kinin zaf & $\mathrm{T}$ & AM191 \\
\hline Mimusops kummel A.DC. & Sapotaceae & Qolati & $\mathrm{T}$ & AM120 \\
\hline Moraea schimperi (Hochst.) Pic.-Serm. & Iridaceae & Loga & S & AM115 \\
\hline Myrsine africana $\mathrm{L}$. & Myrsinaceae & Qachamo & S & AM203 \\
\hline Myrsine melanophoeos (L.) R. Br. & Myrsinaceae & Tuullaa & $\mathrm{T}$ & AM074 \\
\hline Nepeta azurea R.Br. ex Benth. & Lamiaceae & - & $S$ & AM003 \\
\hline Ocotea kenyensis (Chiov.) Robyns \& Wilczek & Lauraceae & Gigicha & $\mathrm{T}$ & AM118 \\
\hline Oldenlandia herbacea (L.) Roxb. & Rubiaceae & Omachessaa & $\mathrm{H}$ & AM028 \\
\hline Olea capensis L.ssp. macrocarpa (C.H.Wright)Verdc. & Oleaceae & Gagama & $\mathrm{T}$ & AM132 \\
\hline Olea europaea L. subsp. cuspidata (Wall.ex G.Don) & Oleaceae & Ejerssaa & $\mathrm{T}$ & AM157 \\
\hline Olea welwitschii (Knobl.) Gilg. \& Schellenb. & Oleaceae & Onomaa & $\mathrm{T}$ & AM134 \\
\hline Osyris compressa (P.J.Bergius) A.DC. & Santalaceae & Waatoo & $\mathrm{S}$ & AM183 \\
\hline Osyris quadripartita Decne. & Santalaceae & Karo & $\mathrm{S}$ & AM198 \\
\hline Pentaschistis minor (Ballard \& C.E.Hubb.) Ballard \& C.E.Hubb. & Poaceae & - & $\mathrm{H}$ & AM061 \\
\hline Phytolacca dodecandra L'Herit. & Phytolaccaceae & Handode & $\mathrm{H}(\mathrm{clim})$ & AM205 \\
\hline Piliostigma thonningii (Schum.) & Fabaceae & Lilluu & $\mathrm{T}$ & AM165 \\
\hline Plantago africana Verdc. & Plantaginaceae & Qinxaa, Baallee & $\mathrm{H}$ & AM045 \\
\hline Podocarpus falcatus (Thunb) C.N & Podocarpaceae & Birbirssaa & $\mathrm{T}$ & AM130 \\
\hline Poecilostachys oplismeoides (Hack.) W.D.Clayton & Poaceae & Daaffa & $\mathrm{H}$ & AM144 \\
\hline Polygala steudneri Chod. & Polygalaceae & Grisa/Garasita & $\mathrm{H}$ & AM005 \\
\hline Polyscias fulva (Hiern) Harms & Araliaceae & Kooribaa & $\mathrm{T}$ & AM139 \\
\hline Polystichum ammifolium (Poir.) C.Chr. & Dryopteridaceae & Qumbuta, Gammanyee & $\mathrm{H}$ & AM069 \\
\hline Pouteria adolfi-friederici (Engl.) Baehni & Sapotaceae & Guduba & $\mathrm{T}$ & AM138 \\
\hline Pseudognaphalium luteo-album (L.) Hilliard and Burtt & Asteraceae & - & $\mathrm{H}$ & AM070 \\
\hline Psidium guajava $\mathrm{L}$ & Myrtaceae & Zeytuna & S & AM177 \\
\hline Psychotria orophila Petit & Rubiaceae & Ulaagaa & $\mathrm{S}$ & AM154 \\
\hline Psydrax schimperiana Spermacoce L. & Rubiaceae & Galle & $\mathrm{T}$ & AM149 \\
\hline Pteris confusa (Lansgd \& Fisch.) Kuhn & Pteridaceae & Qumbuta & $\mathrm{H}$ & AM126 \\
\hline Ranunculus multifidus Forssk. & Ranunculaceae & Sherif & $\mathrm{H}$ & AM077 \\
\hline Rapanea melanophloeos (L.) Mez & Myrsinaceae & Tulla & $\mathrm{T}$ & AM196 \\
\hline Rhus natalensis (Bernh. ex Krauss) F.A.Barkley & Anacardiaceae & Dabaqaa & $\mathrm{S}$ & AM171 \\
\hline Ricinus communis L. & Euphorbiaceae & Koboo, Gulo & S & AM179 \\
\hline Rosa abyssinica Lindley & Rosaceae & Gora & S & AM093 \\
\hline Rubus erlangeri Engl. & Rosaceae & Hato & S & AM004 \\
\hline Rubus steudneri Schwienf. & Rosaceae & Gora & $\mathrm{S}$ & AM086 \\
\hline Rumex abyssinicus Jacq. & Polygonaceae & Shabee Haga & $\mathrm{H}$ & AM050 \\
\hline Rumex nepalensis Spreng. & Polygonaceae & Shabee & $\mathrm{H}$ & AM021 \\
\hline Rytidosperma subulata (A. Rich.) Cope & Poaceae & Marga Hori, Qecha & $\mathrm{H}$ & AM110 \\
\hline Salvia merjame Forssk. & Lamiaceae & Okotu & $\mathrm{S}$ & AM015 \\
\hline Salvia nilotica Jacq. & Lamiaceae & Okotu & $\mathrm{H}$ & AM030 \\
\hline Sanicula elata Buch. -Ham. ex D.Don & Apiaceae & Galee Simbira, Sidissa & $\mathrm{H}$ & AM079 \\
\hline Satureja simensis (Benth.) Briq. & Lamiaceae & Toshimbata & $\mathrm{H}$ & AM049 \\
\hline
\end{tabular}


TABLE 2: Continued.

\begin{tabular}{|c|c|c|c|c|}
\hline Scientific name & Family & Local name (Or.) & Habit & Coll. no. \\
\hline Scabiosa columbaria L. & Dipsacaceae & Anamuro & $\mathrm{H}$ & AM067 \\
\hline Schefflera abyssinica Forst. \& Forst. f., & Araliaceae & Gatamee & $\mathrm{T}$ & AM136 \\
\hline Schefflera volkensii (Engl.) Harms & Araliaceae & Ansha & $\mathrm{T}$ & AM204 \\
\hline Schinus molle L. & Anacardiaceae & Qondabarbere & $\mathrm{T}$ & AM166 \\
\hline Senecio ochrocarpus Oliv. and Hiern & Asteraceae & Agadena & $\mathrm{H}$ & AM046 \\
\hline Senecio ragazzii Chiov. & Asteraceae & Agadena & $\mathrm{H}$ & AM089 \\
\hline Senecio schultzii Hochst. ex A.Rich. & Asteraceae & - & $\mathrm{H}$ & AM057 \\
\hline Setaria megaphylla (Steud.) T.Durand \& Schinz. & Poaceae & Sookora & $\mathrm{H}$ & AM127 \\
\hline Solanum anguivi Lam. & Solanaceae & Mujule Worabessa & $\mathrm{S}$ & AM111 \\
\hline Solanum garae Friis & Solanaceae & - & S & AM085 \\
\hline Solanum marginatum L.f. & Solanaceae & Hidii & S & AM076 \\
\hline Spathodea campanulata (S.nilotica) & Bignoniaceae & Horoqa & $\mathrm{T}$ & AM182 \\
\hline Sporobolus africanus (Poir.) Robyns and Tournay & Poaceae & Marga Hilensa (Or) & $\mathrm{H}$ & AM088 \\
\hline Sporobolus pyramidalis P.Beauv. & Poaceae & Chita & $\mathrm{H}$ & AM124 \\
\hline Stellaria sennii Chiov. & Caryophyllaceae & Duqushu, Dinbiba & $\mathrm{H}$ & AM108 \\
\hline Strychnos mitis S. Moore & Loganiaceae & Muluqaa & $\mathrm{T}$ & AM133 \\
\hline Swertia lugardae Bullock & Gentianaceae & - & $\mathrm{H}$ & AM053 \\
\hline Syzygium guineense (Willd.) DC. & Myrtaceae & Badeesa & $\mathrm{T}$ & AM117 \\
\hline Teclea nobilis Del. & Rutaceae & Hadheessa & $\mathrm{T}$ & AM184 \\
\hline Thymus schimperi Ronniger & Lamiaceae & Tossigne & $\mathrm{H}$ & AM047 \\
\hline Trema orientalis (L.) Bl. & Ulmaceae & Tala’aa & $\mathrm{T}$ & AM188 \\
\hline Trifolium acaule Steud. ex A.Rich. & Fabaceae & - & $\mathrm{H}$ & AM059 \\
\hline Trifolium rueppellianum Fresen. & Fabaceae & Sidissa (Maget) & $\mathrm{H}$ & AM092 \\
\hline Trifolium semipilosum Fresen. & Fabaceae & Sidissa & $\mathrm{H}$ & AM019 \\
\hline Trifolium simense Fresen. & Fabaceae & - & $\mathrm{H}$ & AM034 \\
\hline Trifolium substerraneum L. & Fabaceae & Sidisa (O), Alfalfa(E) & $\mathrm{H}$ & AM038 \\
\hline Triumfetta pentandra A. Rich & Malvaceae & Gurbii & $\mathrm{H}(\operatorname{clim})$ & AM125 \\
\hline Ursinia nana DC. & Asteraceae & Qinxxa & $\mathrm{H}$ & AM022 \\
\hline Urtica dioecia $\mathrm{L}$. & Urticaceae & Dobi(Or), Sama(Amh) & S & AM158 \\
\hline Urtica simensis Steudel & Urticaceae & Dobii & $\mathrm{H}$ & AM109 \\
\hline Vepris dainellii (Pichi-Serm.) Kokwaro & Rutaceae & Arabe & $\mathrm{T}$ & AM129 \\
\hline Vernonia amygdalina Del. & Asteraceae & Ebicha & S & AM192 \\
\hline Vernonia auriculiferaHiern. & Asteraceae & Rejii & $\mathrm{S}$ & AM193 \\
\hline Warburgia ugandensis Sprague & Canellaceae & Bifi, kanafa & $\mathrm{T}$ & AM142 \\
\hline Zehneria scabra (Linn.f.) Sond. & Cucurbitaceae & Harola & $\mathrm{H}(\mathrm{clim})$ & AM096 \\
\hline Ziziphus abyssinicaA.Rich. & Rhamnaceae & Kankura & S & AM195 \\
\hline
\end{tabular}

H, herb; S, shrub; T, tree; Li, liana; H (clim), herbaceous climber; E, epiphyte; PH, parasitic herb; Or., Oromifa; Coll. no., collection number.

and greater canopy openness [56]. Moreover, the seedlings are most affected by edge effect due to their sensitivity to environmental changes and biotic interactions [57, 58]. Conversely, the mean basal area of woody species in the interior habitat $\left(11.16 \pm 1.82 \mathrm{~m}^{2} \mathrm{ha}^{-1}\right)$ was significantly higher than the edge habitat $\left(3.99 \pm 0.54 \mathrm{~m}^{2} \mathrm{ha}^{-1}\right)$ at $p<0.05$ (Figure 2(b); Tables 4 and 5). This was due to the significantly higher mean DBH $(78.62 \pm 4.56 \mathrm{~cm}, p<0.001)$ and height $(33.63 \pm 2.71 \mathrm{~m}, p<0.05)$ of woody species [59] in the interior habitat than the edge. There were $27.32 \%$ of larger diameter individual tree species with $\mathrm{DBH}>100 \mathrm{~cm}$ recorded in the interior habitat, whereas $4.09 \%$ of individuals with $\mathrm{DBH}>100 \mathrm{~cm}$ were identified in the edge habitat. Microenvironmental conditions such as high temperature, low relative humidity, high wind force, low soil nutrient, and litter moisture in the edge habitats may contribute to the changes in tree abundance and distribution in the forest $[60,61]$.

Juniperus procera was the dominant woody species in the edge habitat with an IVI of 32.49, whereas Croton macrostachyus was dominant in the interior habitat with an IVI of
TABle 3: Mean ( \pm SE) values of species richness and diversity indices.

\begin{tabular}{lcc}
\hline Diversity indices & Edge habitat & Interior habitat \\
\hline Species richness $(S)$ & $35 \pm 4.2^{\mathrm{a}}$ & $29 \pm 3.6^{\mathrm{b}}$ \\
Simpson index $(D)$ & $0.10 \pm 0.02$ & $0.11 \pm 0.03$ \\
Shannon-Wiener index $\left(H^{\prime}\right)$ & $2.93 \pm 0.17^{\mathrm{a}}$ & $2.43 \pm 0.11^{\mathrm{b}}$ \\
Pielou's evenness index $\left(J^{\prime}\right)$ & $0.79 \pm 0.05$ & $0.83 \pm 0.04$ \\
Whittaker $\beta$-diversity $\left(\beta_{w}\right)$ & $1.83 \pm 0.26$ & $1.34 \pm 0.31$ \\
Margalef index $\left(D_{M}\right)$ & $5.68 \pm 0.69^{\mathrm{a}}$ & $3.72 \pm 0.92^{\mathrm{b}}$ \\
Berger-Parker index $(d)$ & $0.19 \pm 0.03$ & $0.24 \pm 0.04$ \\
\hline
\end{tabular}

Note. Values with different letters indicate significant differences between habitats $(p=0.05)$.

40.61 (Tables 5 and 6). Accordingly, Juniperus procera, Hagenia abyssinica, and Syzygium guineense were identified as generalists that abundantly occurred in both edge and interior habitats, whereas Hypericum revolutum Vahl. was identified as marginalized species that characteristically dominated the edge habitats $[62,63]$. However, no woody species was found as a specialist that typically occurred in the interior habitats. 


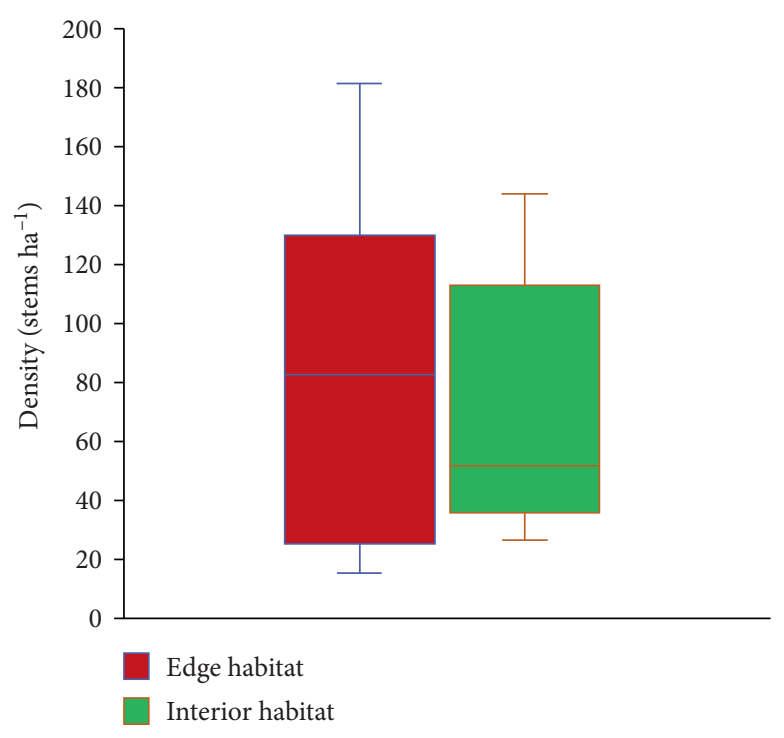

(a)

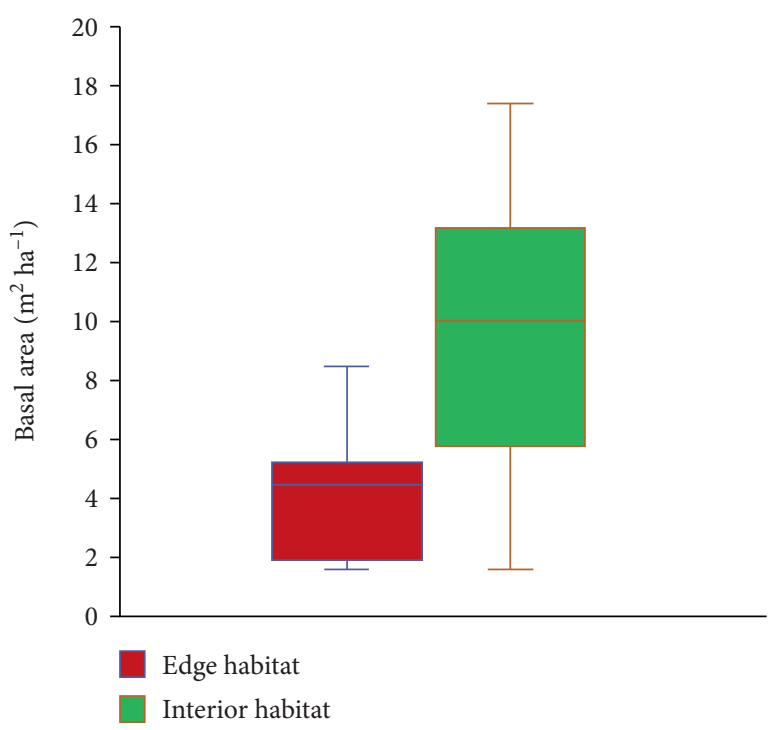

(b)

Figure 2: Box plot showing woody species density (a) and basal area (b) in the habitats.

TABLE 4: Woody species structure in the edge habitats of the BMNP.

\begin{tabular}{|c|c|c|c|c|c|c|c|}
\hline Species & Abundance & $\begin{array}{c}\text { Basal area }\left(\mathrm{m}^{2}\right. \\
\left.\mathrm{ha}^{-1}\right)\end{array}$ & $\begin{array}{c}\text { Density (stem } \\
\mathrm{ha}^{-1} \text { ) }\end{array}$ & $\begin{array}{c}\text { Relative } \\
\text { density }\end{array}$ & $\begin{array}{l}\text { Relative } \\
\text { frequency }\end{array}$ & $\begin{array}{c}\text { Relative } \\
\text { dominance }\end{array}$ & IVI \\
\hline Celtis africana & 12 & 1.94 & 9 & 1.79 & 6.35 & 5.18 & 13.32 \\
\hline Cordia africana & 10 & 4.15 & 8 & 1.49 & 4.76 & 14.81 & 21.06 \\
\hline Croton macrostachyus & 22 & 2.34 & 17 & 3.28 & 6.35 & 6.27 & 15.90 \\
\hline Diospyros abyssinica & 3 & 0.95 & 2 & 0.45 & 1.59 & 5.08 & 7.12 \\
\hline Ehretia cymosa & 12 & 7.31 & 9 & 1.79 & 4.76 & 15.64 & 22.19 \\
\hline Hagenia abyssinica & 32 & 6.84 & 25 & 4.78 & 7.94 & 14.62 & 27.34 \\
\hline Hypericum revolutum & 174 & 1.93 & 136 & 25.97 & 11.11 & 2.95 & 40.03 \\
\hline Juniperus procera & 90 & 6.63 & 70 & 13.43 & 12.70 & 8.87 & 35.00 \\
\hline $\begin{array}{l}\text { Lepidotrichilia } \\
\text { volkensii }\end{array}$ & 4 & 0.48 & 3 & 0.60 & 3.17 & 2.59 & 6.36 \\
\hline Macaranga capensis & 6 & 0.44 & 5 & 0.90 & 3.17 & 2.34 & 6.41 \\
\hline Maytenus undata & 54 & 0.00 & 42 & 8.06 & 4.76 & 0.00 & 12.82 \\
\hline Myrsine melanophoeos & 170 & 0.28 & 133 & 25.37 & 7.94 & 0.61 & 33.92 \\
\hline Olea capensis & 4 & 1.08 & 3 & 0.60 & 4.76 & 5.78 & 11.14 \\
\hline Olea welwitschii & 6 & 0.17 & 5 & 0.90 & 1.59 & 1.79 & 4.27 \\
\hline Podocarpus falcatus & 40 & 0.68 & 31 & 5.97 & 12.70 & 1.21 & 19.88 \\
\hline $\begin{array}{l}\text { Pouteria adolfi- } \\
\text { friederici }\end{array}$ & 3 & 0.19 & 2 & 0.45 & 1.59 & 2.01 & 4.05 \\
\hline Syzygium guineense & 28 & 6.70 & 22 & 4.18 & 4.76 & 10.24 & 19.18 \\
\hline Total & 670 & 42.13 & 523 & 100 & 100 & 100 & 300 \\
\hline
\end{tabular}

IVI: importance value index.

\subsection{Effects of Landscape Change in Floristic Composition and} Structure. The computed regression analysis among the landscape indices and species composition and structural parameters in this study revealed that only $\mathrm{PN}$ and AREA_MN significantly affected both the species composition and structural properties of the study area. Accordingly, PN was strong and negatively affected the overall species richness $(r=-0.90, p<0.05)$ and Shannon diversity index $(r=-0.96, p<0.01)$ (Table 7$)$. Conversely, the overall species richness $(r=0.95, p<0.05)$ and Shannon diversity $(r=0.87, \quad p<0.05)$ have shown strong and positive correlation with AREA_MN. This implies that as the number of fragmented habitats increases, species richness and diversity, particularly interior-dependent species, decreases. However, edge-dependent species comfortably flourished. One of the consequences of habitat fragmentation is an increase in the proportional abundance of the edge influenced habitat and its adverse impacts on interior-sensitive species [64]. Undoubtedly, while some species (e.g., habitat specialists) suffer from fragmentation, others benefit from it (e.g., generalists and edge species) [65]. Consequently, PN was strong and negatively correlated with AREA_MN 
TABLE 5: Woody species structure in the interior habitats of the BMNP.

\begin{tabular}{|c|c|c|c|c|c|c|c|}
\hline Species & Abundance & $\begin{array}{c}\text { Basal area }\left(\mathrm{m}^{2}\right. \\
\left.\mathrm{ha}^{-1}\right)\end{array}$ & $\begin{array}{c}\text { Density (stem } \\
\mathrm{ha}^{-1} \text { ) }\end{array}$ & $\begin{array}{l}\text { Relative } \\
\text { density }\end{array}$ & $\begin{array}{l}\text { Relative } \\
\text { frequency }\end{array}$ & $\begin{array}{c}\text { Relative } \\
\text { dominance }\end{array}$ & IVI \\
\hline Allophylus macrobotrys & 6 & 0.71 & 5 & 0.79 & 2.86 & 1.76 & 5.41 \\
\hline Celtis africana & 16 & 0.25 & 13 & 2.12 & 3.81 & 0.46 & 6.39 \\
\hline Croton macrostachyus & 142 & 8.64 & 111 & 18.81 & 6.67 & 9.15 & 34.62 \\
\hline Diospyros abyssinica & 4 & 0.08 & 3 & 0.53 & 1.90 & 0.58 & 3.01 \\
\hline Ehretia cymosa & 74 & 0.83 & 58 & 9.80 & 4.76 & 2.06 & 16.62 \\
\hline Erica arborea & 3 & 0.02 & 2 & 0.45 & 1.59 & 0.17 & 2.20 \\
\hline $\begin{array}{l}\text { Elaeodendron } \\
\text { buchananii }\end{array}$ & 18 & 0.42 & 14 & 2.38 & 4.76 & 0.62 & 7.77 \\
\hline Fagaropsis angolensis & 3 & 0.02 & 2 & 0.40 & 0.95 & 0.18 & 1.53 \\
\hline Hagenia abyssinica & 40 & 10.43 & 31 & 5.30 & 7.62 & 9.67 & 22.58 \\
\hline Hypericum revolutum & 8 & 0.05 & 6 & 1.06 & 6.67 & 0.18 & 7.90 \\
\hline Juniperus procera & 128 & 31.54 & 100 & 16.95 & 7.62 & 29.24 & 53.81 \\
\hline Lepidotrichilia volkensii & 4 & 0.01 & 3 & 0.53 & 0.95 & 0.11 & 1.59 \\
\hline Macaranga capensis & 6 & 0.03 & 5 & 0.79 & 1.90 & 0.12 & 2.82 \\
\hline Margaritaria discoidea & 4 & 0.03 & 3 & 0.53 & 1.90 & 0.13 & 2.56 \\
\hline Maytenus undata & 49 & 0.06 & 38 & 6.49 & 2.86 & 0.16 & 9.50 \\
\hline Mimusops kummel & 3 & 0.04 & 2 & 0.40 & 0.95 & 0.27 & 1.62 \\
\hline Myrsine melanophoeos & 32 & 0.10 & 25 & 4.24 & 1.90 & 0.37 & 6.51 \\
\hline Ocotea kenyensis & 32 & 1.13 & 25 & 4.24 & 6.67 & 1.20 & 12.10 \\
\hline Olea europaea & 8 & 0.72 & 6 & 1.06 & 1.90 & 1.77 & 4.73 \\
\hline Olea welwitschii & 6 & 0.17 & 5 & 0.79 & 2.86 & 1.24 & 4.89 \\
\hline Podocarpus falcatus & 78 & 6.66 & 61 & 10.33 & 7.62 & 6.18 & 24.13 \\
\hline Polyscias fulva & 3 & 0.24 & 2 & 0.40 & 0.95 & 1.79 & 3.14 \\
\hline $\begin{array}{l}\text { Pouteria adolfi- } \\
\text { friederici }\end{array}$ & 8 & 2.61 & 6 & 1.06 & 3.81 & 4.83 & 9.70 \\
\hline Psydrax schimperiana & 4 & 0.08 & 3 & 0.53 & 1.90 & 0.31 & 2.74 \\
\hline Schefflera abyssinica & 3 & 0.20 & 2 & 0.40 & 0.95 & 1.49 & 2.84 \\
\hline Strychnos mitis & 28 & 0.28 & 22 & 3.71 & 3.81 & 0.52 & 8.04 \\
\hline Syzygium guineense & 35 & 9.55 & 27 & 4.64 & 6.67 & 23.60 & 34.90 \\
\hline Vepris dainellii & 4 & 0.25 & 3 & 0.53 & 1.90 & 0.94 & 3.37 \\
\hline Warburgia ugandensis & 6 & 0.25 & 5 & 0.79 & 1.90 & 0.94 & 3.64 \\
\hline Total & 755 & 75.41 & 590 & 100 & 100 & 100 & 300 \\
\hline
\end{tabular}

IVI: importance value index.

TABLE 6: IVI of woody species in the edge and interior habitats.

\begin{tabular}{|c|c|c|c|c|c|c|c|c|}
\hline \multirow[t]{2}{*}{ Species } & \multicolumn{2}{|c|}{ Relative density (\%) } & \multicolumn{2}{|c|}{$\begin{array}{l}\text { Relative frequency } \\
(\%)\end{array}$} & \multicolumn{2}{|c|}{$\begin{array}{l}\text { Relative dominance } \\
(\%)\end{array}$} & \multicolumn{2}{|c|}{ IVI } \\
\hline & Edge & Interior & Edge & Interior & Edge & Interior & Edge & Interior \\
\hline Juniperus procera & 11.72 & 20.13 & 8.79 & 11.94 & 11.98 & 25.03 & 32.49 & 37.10 \\
\hline Croton macrostachyus & 2.86 & 22.33 & 4.40 & 10.45 & 8.47 & 7.83 & 15.73 & 40.61 \\
\hline Hagenia abyssinica & 5.21 & 5.03 & 5.49 & 11.94 & 19.76 & 8.28 & 30.47 & 25.25 \\
\hline Syzygium guineense & 3.65 & 5.35 & 7.69 & 4.48 & 13.84 & 20.21 & 25.18 & 30.03 \\
\hline Podocarpus falcatus & 5.21 & 12.26 & 6.59 & 11.94 & 1.64 & 5.29 & 13.44 & 29.49 \\
\hline Myrsine melanophoeos & 22.14 & 5.03 & 5.49 & 2.99 & 0.82 & 0.31 & 28.45 & 8.33 \\
\hline Ehretia cymosa & 1.56 & 11.64 & 3.30 & 7.46 & 4.01 & 9.29 & 8.87 & 28.38 \\
\hline Hypericum revolutum & 22.66 & - & 7.69 & - & 3.99 & - & 34.34 & - \\
\hline Cordia africana & 1.57 & - & 4.48 & - & 8.79 & - & 14.84 & - \\
\hline Ocotea kenyensis & 4.17 & - & 7.69 & - & 2.33 & - & 14.19 & - \\
\hline
\end{tabular}

$(r=-0.71, p<0.001)$. This implies that as the PN increases, the area of fragments decreases; as a result, small fragments contain a smaller species richness and lower species density than large fragments [66]. Large areas of habitat tend to support more individuals and, hence, more species [67].

Besides, modifying the spatial pattern of the landscape habitat size reduction and increase in isolation cause an alteration in the dispersal rate, affecting survival and mortality of individuals [8]. Many population and community changes in habitat fragments were commonly attributed to edge effects [66]. Interior species may be affected by the size decrease in their habitat, by edge effect, and by competition with generalists $[68,69]$. The most threatened endemic species due to edge effect in the BMNP were Helichrysum harennense Mesfin, Kniphofia insignis Rendle, Rubus erlangeri Engl., and Vepris dainellii Pichi Serm 
TABLE 7: Pearson's correlation coefficient between the landscape indices and floristic composition and structural parameters.

\begin{tabular}{|c|c|c|c|c|c|c|c|c|c|c|c|}
\hline$S$ & 1 & & & & & & & & & & \\
\hline$H^{\prime}$ & $0.95^{*}$ & 1 & & & & & & & & & \\
\hline $\mathrm{BA}$ & $0.96^{*}$ & $0.94^{*}$ & 1 & & & & & & & & \\
\hline Density & $0.99^{* *}$ & $0.90^{*}$ & $0.93^{*}$ & 1 & & & & & & & \\
\hline $\mathrm{PN}$ & $-0.90^{*}$ & $-0.96^{*}$ & $-0.96^{* *}$ & $-0.84^{*}$ & 1 & & & & & & \\
\hline AREA_MN & $0.95^{*}$ & $0.87^{*}$ & $0.82^{*}$ & $0.72^{*}$ & $-0.71^{* * *}$ & 1 & & & & & \\
\hline SHAPE_AM & -0.42 & -0.64 & -0.53 & -0.28 & $0.48^{*}$ & -0.01 & 1 & & & & \\
\hline $\mathrm{COA}$ & -0.56 & -0.37 & 0.34 & 0.66 & $-0.87^{*}$ & 0.41 & $-0.79^{* * *}$ & 1 & & & \\
\hline ED & 0.65 & 0.78 & -0.77 & -0.54 & $0.54^{*}$ & -0.91 & 0.25 & -0.16 & 1 & & \\
\hline ENN_MN & -0.37 & -0.56 & -0.59 & -0.28 & $0.77^{* * *}$ & -0.26 & $0.73^{* *}$ & $-0.81^{* * *}$ & 0.04 & 1 & \\
\hline \multirow[t]{2}{*}{ IJI } & -0.42 & -0.28 & -0.32 & -0.37 & $0.58^{*}$ & $-0.59^{* *}$ & 0.37 & -0.61 & 0.37 & 0.1732 & 1 \\
\hline & S & $H^{\prime}$ & BA & Density & PN & AREA_MN & SHAPE_AM & $\mathrm{COA}$ & ED & ENN_MN & IJI \\
\hline
\end{tabular}

Note. The star indicates significant level between values $\left({ }^{*} p<0.05,{ }^{* *} p<0.01\right.$, and $\left.{ }^{* * *} p<0.001\right)$.

Kokwaro. Also, the most common invasive species in the study area favored by edge effect was Achyranthes aspera L, which is also common in the disturbed forests and forest edges of the dry Afromontane forests and moist Afromontane forests in Ethiopia [70]. The gradual decline in the more sensitive species may induce a species turnover in fragments and cascade effects $[62,63]$.

Among the landscape indices computed, only PN and AREA_MN significantly affected some of the floristic structural properties assessed. Thus, the PN was strong and negatively affected the woody species density $(r=-0.84$, $p<0.05)$ and basal area $(r=-0.96, p<0.01)$, as well as AREA_MN was strong and positively affected the density $(r=0.71, p<0.05)$ and basal area $(r=0.82, p<0.05)$ of woody species. Habitat destruction, isolation, and transformation affect the structure and dynamics of populations, communities, and ecosystems, as well as ecological processes [71]. Generally, as AREA_MN and COA of patches increase, species richness, diversity, evenness, woody species density, basal area, DBH, and height also increase. However, as PN, SHAPE_MN, ED, ENN_MN, and IJI of patches increase, floristic composition and structural variables decrease. This implies that the landscape composition and configuration change may potentially affect the vegetation composition and structure of a particular area.

\section{Conclusion}

This study recognized that the Bale Mountains National Park has a diverse biodiversity and is an ecologically significant area. It contains a variety of life forms with good ecological integration. It also harbors a number of endemic floras and faunas. However, currently, anthropogenic disturbances strongly impaired the plant species composition and structure as well as the overall ecological integrity of the landscape. The progressive settlement and agricultural land expansion at the expense of natural forest and grassland coupled with human-induced recurrent fire and livestock grazing in park became a potential threat to the landscape structure. This was due to the escalated human and livestock population and their corresponding demands and necessities increment in the park. Both the floristic composition and structure were affected by the expansion of edge habitat and shrinkage of interior habitat. Species richness and diversity were higher in the edge habitat, whereas density, frequency, and basal area were higher in the interior habitat. Therefore, maintenance of the habitats heterogeneity in the park is essential for long-term population persistence. Moreover, human activities in the park should be banned and settlements in the park should be relocated to other areas to avoid their potential impacts on the floras and faunas. Finally, studies on microenvironmental factors such as light availability, air and soil temperature, humidity, and soil nutrients along the edge and interior gradient should be conducted to determine their effect on species richness, composition, and structure.

\section{Data Availability}

The data used to support the findings of this study are included within this paper.

\section{Conflicts of Interest}

The authors declare that there are no conflicts of interest.

\section{Acknowledgments}

The authors would like to express their special thanks to the Ethiopian Wildlife Authority for giving permission to conduct this study on-site.

\section{References}

[1] D. B. Lindenmayer and J. Fischer, Habitat Fragmentation and Landscape Change: An Ecological and Conservation Synthesis, Island Press, Washington, DC, USA, 2013.

[2] D. Mullu, "A review on the effect of habitat fragmentation on ecosystem," Journal of Natural Sciences Research, vol. 6, no. 15, pp. 1-15, 2016.

[3] L. Gibson, A. J. Lynam, C. J. A. Bradshaw et al., "Nearcomplete extinction of native small mammal fauna 25 years after forest fragmentation," Science, vol. 341, no. 6153, pp. 1508-1510, 2013.

[4] M. Krosby, J. Tewksbury, N. M. Haddad, and J. Hoekstra, "Ecological connectivity for a changing climate," Conservation Biology, vol. 24, no. 6, pp. 1686-1689, 2010. 
[5] H. M. Pereira, P. W. Leadley, V. Proença et al., "Scenarios for global biodiversity in the 21st century," Science, vol. 330, no. 6010, pp. 1496-1501, 2010.

[6] J. E. Rogan and T. E. Lacher Jr, Impacts of Habitat Loss and Fragmentation on Terrestrial Biodiversity, Elsevier, Amsterdam, Netherlands, 2018.

[7] N. M. Haddad, L. A. Brudvig, J. Clobert et al., "Habitat fragmentation and its lasting impact on Earth's ecosystems," Science Advances, vol. 1, no. 2, Article ID e1500052, 2015.

[8] L. Fahrig, "Effects of habitat fragmentation on biodiversity," Annual Review of Ecology, Evolution, and Systematics, vol. 34, no. 1, pp. 487-515, 2003.

[9] A. F. Bennett and D. A. Saunders, "Habitat fragmentation and landscape change," Conservation Biology, vol. 93, pp. 15441550, 2010.

[10] J. L. Hill and P. J. Curran, "Species composition in fragmented forests: conservation implications of changing forest area," Applied Geography, vol. 21, no. 2, pp. 157-174, 2001.

[11] K. Aliyi, K. Hundera, and G. Dalle, "Floristic composition, vegetation structure and regeneration status of kimphe lafa natural forest, oromia regional state, West Arsi, Ethiopia," Research \& Reviews: A Journal of Life Sciences, vol. 5, no. 1, pp. 19-32, 2015.

[12] D. Vergara-Rodríguez, G. Mathieu, M.-S. Samain, S. Armenta-Montero, and T. Krömer, "Diversity, distribution, and conservation status of Peperomia (Piperaceae) in the state of Veracruz, Mexico," Tropical Conservation Science, vol. 10, 2017.

[13] A. Ahmedin and E. Elias, "Tree species composition, structure and regeneration status in Munessa natural forest, Southeastern Ethiopia," Eurasian Journal of Forest Science, vol. 8, no. 1, pp. 35-53, 2020.

[14] F. Yimer, Soil Properties in Relation to Topographic Aspects, Vegetation Communities and Land Use in the South-Eastern Highlands of ETHIOPIA, Swedish University of Agricultural Sciences, vol. 2007, no. 45, Uppsala, Sweden, 2007.

[15] (Woody Biomass Inventory and Strategic Planning Project) WBISPP, A National Strategic Plan for the Biomass Energy Sector, Ministry of Agriculture, Addis Ababa, Ethiopia, 2004.

[16] K. Belay, "Resettlement of peasants in Ethiopia," Journal of Rural Development, vol. 27, pp. 223-253, 2004.

[17] J. W. Mellor and P. Dorosh, "Agriculture and the economic transformation of Ethiopia," in Proceedings of the ESSP II Working Paper, Washington, DC, USA, 2010.

[18] A. Mekonnen, A. Bekele, G. Hemson, E. Teshome, and A. Atickem, "Population size and habitat preference of the vulnerable Bale monkey Chlorocebus djamdjamensis in odobullu forest and its distribution across the Bale Mountains, Ethiopia," Oryx, vol. 44, no. 4, pp. 558-563, 2010.

[19] P. A. Stephens, C. A. d'Sa, C. Sillero-Zubiri, and N. LeaderWilliams, "Impact of livestock and settlement on the large mammalian wildlife of Bale Mountains National Park, Southern Ethiopia," Biological Conservation, vol. 100, no. 3, pp. 307-322, 2001.

[20] K. B. G. M. S. Tesfaye and Z. Bires, "Awareness creation at community level towards the conservation of cultural and natural tourism resources of Bale Mountains National Park and Harena Buluk, South East Ethiopia," Journal of Hospitality, Leisure, Sport \& Tourism Educationa, vol. 1, p. 14, 2015.

[21] G. Williams-Linera, "Tree species richness complementarity, disturbance and fragmentation in a Mexican tropical montane cloud forest," Biodiversity and Conservation, vol. 11, no. 10, pp. 1825-1843, 2002.
[22] OARDB, "Bale mountains national park general management plan," OARDB, Addis Ababa, Ethiopia, 2007.

[23] J. C. Hillman, "The Bale mountains national park area, Southeast Ethiopia, and its management," Mountain Research and Development, vol. 8, no. 2/3, pp. 253-258, 1988.

[24] S. Miehe and G. Miehe, "Ericaceous forests and heathlands in the Bale Mountains of South Ethiopia," in Ecology and Man's Impact, Hamburg University Press, Hamburg, Germany, 1994.

[25] R. G. Barry, Mountain Weather and Climate, Cambridge University Press, New York, NY, USA, 2008.

[26] D. D. Daye, Fragmented Forests in South-West Ethiopia: Impacts of Land-Use Change on Plant Species Composition and Priorities for Future Conservation, Prifysgol Bangor University, Bangor, UK, 2012.

[27] S. Edwards, M. Tadesse, S. Demissew, and I. Hedberg, Flora of Ethiopia and Eritrea, Volume 2, Part 1: Magnoliaceae to Flacourtiaceae, Addis Ababa University, Addis Ababa, Ethiopia, 2000.

[28] I. Hedberg, E. Kelbessa, S. Edwards, S. Demissew, and E. Persson, Flora of Ethiopia and Eritrea, Volume 5: Gentianaceae to Cyclocheilaceae, Addis Ababa University, Addis Ababa, Ethiopia, 2006.

[29] A. E. Magurran, Measuring Biological Diversity, John Wiley \& Sons, Hoboken, NY, USA, 2013.

[30] R. H. Økland, Vegetation Ecology: Theory, Method and Applications with Reference to Fennoscandia, Botanical Garden and Museum, University of Oslo, Oslo, Norway, 1990.

[31] D. Ellenberg and D. Mueller-Dombois, Aims and Methods of Vegetation Ecology, Wiley, New York, NY, USA, 1974.

[32] G. J. Martin, Ethnobotany: A Methods Manual, Routledge, London, UK, 2010.

[33] H. Kitessa, B. Tamrat, and K. Ensermu, "Floristic and phytogeographic synopsis of a dry Afromontane coniferous forest in the Bale Mountains (Ethiopia): implications to biodiversity conservation," SINET Ethiopian Journal of Science, vol. 30, pp. 1-12, 2007.

[34] E. Van der Maarel, "Transformation of cover-abundance values in phytosociology and its effects on community similarity," Vegetatio, vol. 39, no. 2, pp. 97-114, 1979.

[35] K. McGarigal, S. A. Cushman, and E. Ene, "FRAGSTATS v4: spatial pattern analysis program for categorical and continuous maps," 2012, http://http//www.\%20umass.\%20edu/ landeco/research/fragstats/fragstats.\%20html.

[36] D. Smiraglia, T. Ceccarelli, S. Bajocco, L. Perini, and L. Salvati, "Unraveling landscape complexity: land use/land cover changes and landscape pattern dynamics (1954-2008) in contrasting Peri-Urban and agro-forest regions of Northern Italy," Environmental Management, vol. 56, no. 4, pp. 916-932, 2015.

[37] K. McGarigal, FRAGSTATS: Spatial Pattern Analysis Program for Categorical Maps, University of Massachusetts, Amherst, MA, USA, 2002, http://http//www.\%20umass.\%20edu/ landeco/research/fragstats/fragstats.\%20html.

[38] Ø. Hammer, D. A. T. Harper, and P. D. Ryan, "PAST: paleontological statistics software package for education and data analysis," Palaeontologia Electronica, vol. 4, no. 1, p. 9, 2001.

[39] B. Oertli, D. A. Joye, E. Castella, R. Juge, D. Cambin, and J.-B. Lachavanne, "Does size matter? The relationship between pond area and biodiversity," Biological Conservation, vol. 104, no. 1, pp. 59-70, 2002. 
[40] B. Flowers, K.-T. Huang, and G. O. Aldana, "Analysis of the habitat fragmentation of ecosystems in Belize using landscape metrics," Sustainability, vol. 12, no. 7, p. 3024, 2020.

[41] Y. Kidane, R. Stahlmann, and C. Beierkuhnlein, "Vegetation dynamics, and land use and land cover change in the Bale Mountains, Ethiopia," Environmental Monitoring and Assessment, vol. 184, no. 12, pp. 7473-7489, 2012.

[42] T. Tolessa, F. Senbeta, and M. Kidane, "The impact of land use/land cover change on ecosystem services in the central highlands of Ethiopia," Ecosystem Services, vol. 23, pp. 47-54, 2017.

[43] M. I. Posada Posada, Using Landscape Pattern Metrics to Characterize Ecoregions, University of Nebraska-Lincoln, Lincoln, NE, USA, 2012.

[44] F. Senbeta, C. Schmitt, T. Woldemariam, H. J. Boehmer, and M. Denich, "Plant diversity, vegetation structure and relationship between plant communities and environmental variables in the Afromontane Forests of Ethiopia," SINET: Ethiopian Journal of Science, vol. 37, no. 2, pp. 113-130, 2014.

[45] A. Dibaba, T. Soromessa, A. Kefalew, and A. Addi, "Woody species diversity, vegetation structure, and regeneration status of the moist afromontane forest of Agama in southwestern Ethiopia," International Journal of Ecology, vol. 2020, Article ID 1629624, 10 pages, 2020.

[46] A. Berhanu, S. Demissew, Z. Woldu, and M. Didita, "Woody species composition and structure of Kuandisha afromontane forest fragment in northwestern Ethiopia," Journal of Forestry Research, vol. 28, no. 2, pp. 343-355, 2016.

[47] G. Fisaha, K. Hundera, and G. Dalle, "Woody plants' diversity, structural analysis and regeneration status of Wof Washa natural forest, North-East Ethiopia," African Journal of Ecology, vol. 51, no. 4, pp. 599-608, 2013.

[48] A. T. Ayalew, Vegetation Ecology and Carbon Stock of WofWasha Forest, Addis Ababa University, Addis Ababa, Ethiopia, 2018.

[49] D. Sebsebe and I. Friis, "Natural vegetation of the Flora area," in Flora of Ethiopia and Eritrea, vol. 8, pp. 27-32, National Herbarium, Addis Ababa University, Addis Ababa, Ethiopia, 2009.

[50] Z. Woldu, M. Fetene, and A. Abate, "Vegetation under different tree species in Acacia woodland in the Rift Valley of Ethiopia," Ecological Studies: Analysis and Synthesis, vol. 22, no. 2, pp. 235-252, 1999.

[51] F. T. Maestre, "On the importance of patch attributes, environmental factors and past human impacts as determinants of perennial plant species richness and diversity in Mediterranean semiarid steppes," Diversity and Distributions, vol. 10, no. 1, pp. 21-29, 2004.

[52] J. W. Ranney, M. C. Bruner, and J. B. Levenson, "Importance of edge in the structure and dynamics of forest islands," Ecological studies: Analysis and Synthesis, vol. 41, 1981.

[53] D. S. Kacholi, "Edge-interior disparities in tree species and structural composition of the Kilengwe forest in Morogoro region, Tanzania," International Scholarly Research Notices, vol. 2014, Article ID 873174, 8 pages, 2014.

[54] W. F. Laurance, L. V. Ferreira, J. M. Rankin-de Merona, and S. G. Laurance, "Rain forest fragmentation and the dynamics of Amazonian tree communities," Ecology, vol. 79, no. 6, pp. 2032-2040, 1998.

[55] J. Benitez-Malvido, "Impact of forest fragmentation on seedling abundance in a tropical rain forest," Conservation Biology, vol. 12, no. 2, pp. 380-389, 1998.

[56] W. F. Laurance, "Forest-climate interactions in fragmented tropical landscapes," Philosophical Transactions of the Royal
Society of London. Series B: Biological Sciences, vol. 359, no. 1443 , pp. 345-352, 2004.

[57] C. Murcia, "Edge effects in fragmented forests: implications for conservation," Trends in Ecology \& Evolution, vol. 10, no. 2, pp. 58-62, 1995.

[58] D. A. Saunders, R. J. Hobbs, and C. R. Margules, "Biological consequences of ecosystem fragmentation: a review," Conservation Biology, vol. 5, no. 1, pp. 18-32, 1991.

[59] H. Yineger, E. Kelbessa, T. Bekele, and E. Lulekal, "Floristic composition and structure of the dry afromontane forest at Bale Mountains National Park, Ethiopia," SINET: Ethiopian Journal of Science, vol. 31, no. 2, pp. 103-120, 2008.

[60] W. D. Newmark, "Tanzanian forest edge microclimatic gradients: dynamic patterns," Biotropica, vol. 33, no. 1, pp. 2-11, 2001.

[61] M. Yan, Z. Zhong, and J. Liu, "Habitat fragmentation impacts on biodiversity of evergreen broadleaved forests in Jinyun Mountains, China," Frontiers of Biology in China, vol. 2, no. 1, pp. 62-68, 2007.

[62] S. L. Pimm, C. N. Jenkins, R. Abell et al., "The biodiversity of species and their rates of extinction, distribution, and protection," Science, vol. 344, no. 6187, 2014.

[63] M. Lomolino and M. D. Weiser, "Towards a more general species-area relationship: diversity on all islands, great and small," Journal of Biogeography, vol. 28, no. 4, pp. 431-445, 2001.

[64] C. S. Robbins, D. K. Dawson, and B. A. Dowell, "Habitat area requirements of breeding forest birds of the middle Atlantic states," Wildlife Monographs, vol. 103, pp. 3-34, 1989.

[65] K. Henle, K. F. Davies, M. Kleyer, C. Margules, and J. Settele, "Predictors of species sensitivity to fragmentation," Biodiversity and Conservation, vol. 13, no. 1, pp. 207-251, 2004.

[66] W. F. Laurance and H. L. Vasconcelos, "Deforestation and forest fragmentation in the amazon," Tropical Biology and Conservation Management, vol. 2, pp. 23-29, 2009.

[67] M. L. Rosenzweig, Species Diversity in Space and Time, Cambridge University Press, Cambridge, UK, 1995.

[68] D. T. Bolger, T. A. Scott, and J. T. Rotenberry, "Use of corridor-like landscape structures by bird and small mammal species," Biological Conservation, vol. 102, no. 2, pp. 213-224, 2001.

[69] C. Schonewald-Cox and M. Buechner, "Park protection and public roads," in Conservation Biology, pp. 373-395, Springer, Berlin, Germany, 1992.

[70] I. Friis, S. Demissew, and P. Van Breugel, Atlas of the potential vegetation of Ethiopia, Det Kongelige Danske Videnskabernes Selskab, Copenhagen, Denmark, 2010.

[71] M. E. Soulé and G. H. Orians, "Conservation biology research: its challenges and contexts," in Conservation Biology: Research Priorities for the Next Decade, pp. 271-285, Springer, Berlin, Germany, 2001. 